\title{
Coupling quantum corrals to form artificial molecules
}

\author{
S.E. Freeney ${ }^{1}$, S.T.P. Borman ${ }^{1}$, J.W. Harteveld ${ }^{1}$ I. Swart ${ }^{1 *}$ \\ 1 Debye Institute for Nanomaterials Science, Utrecht University, the Netherlands * \\ I.Swart@uu.nl
}

August 14, 2020

\begin{abstract}
Quantum corrals can be considered as artificial atoms. By coupling many quantum corrals together, artificial matter can be created at will. The atomic scale precision with which the quantum corrals can be made grants the ability to tune parameters that are difficult to control in real materials, such as the symmetry of the states that couple, the on-site energy of these states, the hopping strength and the magnitude of the orbital overlap. Here, we systematically investigate the accessible parameter space for the dominant platform (CO molecules on $\mathrm{Cu}(111)$ ) by constructing (coupled) quantum corrals of different sizes and shapes. By changing the configuration of the $\mathrm{CO}$ molecules that constitute the barrier between two quantum corrals, the hopping integral can be tuned between 0 and $-0.3 \mathrm{eV}$ for s- and p-like states, respectively. Incorporation of orbital overlap is essential to account for the experimental observations. Our results aid the design of future artificial lattices.
\end{abstract}

\section{Contents}

\begin{tabular}{lll}
\hline & Introduction & $\mathbf{2}$
\end{tabular}

1.1 Tight binding description of dimers and trimers 4

\begin{tabular}{|ll|l}
\hline & Methods & 5
\end{tabular}

\begin{tabular}{lll}
\hline 3 & Results & 6
\end{tabular}

3.1 Individual Corrals 6

\begin{tabular}{lll}
\hline 3.2 & Corral size and on-site energy & 7
\end{tabular}

\begin{tabular}{llr}
\hline 3.3 & Coupling Corrals & 9
\end{tabular}

\begin{tabular}{lll}
\hline 3.4 & Tuning parameters & 10
\end{tabular}

\begin{tabular}{lll}
\hline 3.5 & State selective coupling & 13
\end{tabular}

\begin{tabular}{lll}
\hline 3.6 & Coupling corrals of different sizes & 13
\end{tabular}

\begin{tabular}{lll}
\hline 4 & Conclusion & 14
\end{tabular}

\begin{tabular}{lr}
\hline References & 15
\end{tabular} 


\section{Introduction}

The scanning tunneling microscope makes it possible to position adsorbates and vacancies on surfaces with atomic scale accuracy [1]. This approach has been used to explore the limits of data storage [2 5], to perform logic operations [6 9$]$, to study chemical reactions at the single molecule level [10 13], and to study the electronic and magnetic structure of atomically well-defined structures [14,15].

With respect to studying electronic properties of extended systems, two complementary approaches have been used. The first approach is based on coupling localized states of either adatoms, vacancies or dangling bonds, 16 23. By positioning such species with atomic scale precision, artificial electronic molecules or lattices can be created and their electronic structure studied. Initial experiments focused on the evolution of the electronic structure with system size. However, more complicated and interesting phenomena can also be studied, such as topological states of matter and Majorana bound states 21,24.

The second approach, following the ideas underpinning the quantum corral, is based on patterning a 2D electron gas (2DEG) with a (periodic) scattering potential. In particular, the $\mathrm{CO}$ on $\mathrm{Cu}(111)$ platform has been used to study the electronic structure of periodic and non-periodic systems 25 31. Here, the CO molecules act as repulsive scattering centers for the surface state electrons of $\mathrm{Cu}(111)$ [32]. By placing these scattering centers with atomic scale accuracy, a large variety of potential energy landscapes can be created for electrons. For example, by creating a triangular lattice of $\mathrm{CO}$ molecules, the electrons are confined to the anti-lattice, i.e. a honeycomb geometry [26]. Density of states measurements revealed the emergence of a Dirac cone in the 2DEG, as observed in graphene. Building on this approach, an electronic Lieb-lattice [27], quasi-crystal [28] and electronic fractal [29] have been realized. Recently, it was shown that this material platform can also be used to study topological states of matter [31,33.

One of the advantages of using artificial lattices is that it allows control over parameters that cannot be controlled easily in real materials. These include the on-site energy, the strength of the hopping parameter, orbital overlap, and which orbitals couple. However, the values for the hopping parameter, on-site energy of each electronic site and overlap are not immediately obvious given a certain configuration of $\mathrm{CO} / \mathrm{Cu}(111)$. Currently, determining these parameters is an involved iterative "reverse engineering" procedure which includes first designing the lattice and performing a muffin tin calculation to check that the features of interest are observable, which may take several iterations of design changes. The resulting muffin tin band structure is compared to the output of a tight binding calculation. The tight binding parameters are then adjusted such that the tightbinding band structure matches the muffin tin result 33 .

In this work, we systematically investigate the accessible tight binding parameter range for the $\mathrm{CO} / \mathrm{Cu}(111)$ platform by coupling quantum corrals into artificial molecules. The report is arranged as follows. First, a background on the subject is given, and the experimental details are discussed. We show how changing the size of rectangular and triangular corrals affects their on-site energy and we determine the effective mass of the confined electrons. We specifically focus on rectangular and triangular corrals, as these allow for space-filling artificial lattices. Furthermore, we report experiments on coupling such units into dimers and trimers and extract the tight binding parameters. We investigated the coupling of both $s$-like and $p$-like orbitals. The coupling strength is adjusted with different methods; both by changing the size of the potential barrier between the corrals, and by changing the size of the corrals themselves. Finally, we studied the coupling of orbitals with different symmetries.

Before describing our results, we discuss the similarities and differences between quan- 
(a)

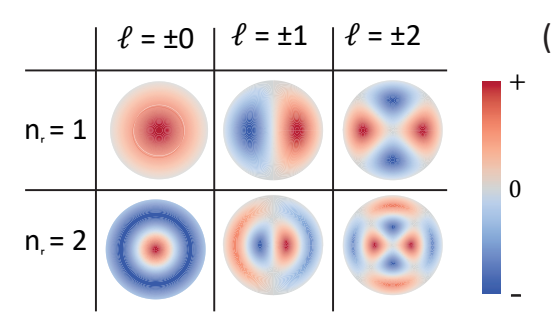

(b)

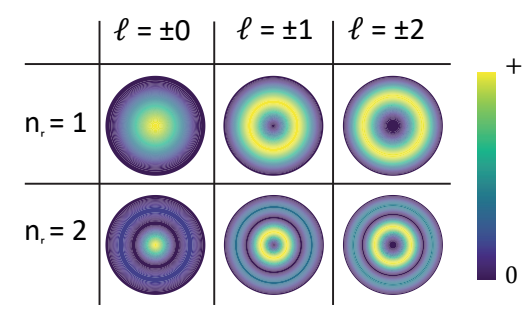

(c) Experimental

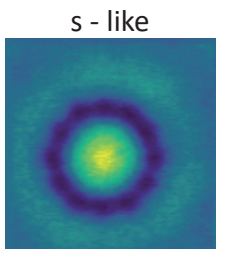

p - like

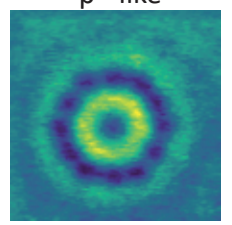

Figure 1: Modeling a circular quantum corral with the particle-in-a-box model. (a) The real part of the wavefunction enclosed in a circular well, showing its shape for different quantum numbers. (b) $|\Psi|^{2}$, which is proportional to a measurement of differential conductance STM. (c) Differential conductance maps of a small quantum corral at two energies; $-0.17 \mathrm{~V}$ and $0.21 \mathrm{~V}$. These correspond to the $\ell=0, n=1(1 s)$ and $\ell=1, n=1(1 p)$ states.

tum corrals and real atoms. Artificial lattices built using $\mathrm{CO} / \mathrm{Cu}(111)$ can be thought of as systems of coupled quantum corrals. The first quantum corral was created by positioning $\mathrm{Fe}$ atoms in a (nearly) circular ring on $\mathrm{Cu}(111)$ [1]. The electronic behavior within the corral can be readily understood in terms of a particle-in-a-box model [1, 34. Fig. 1(a) shows wavefunctions of a particle-in-a-circular-box for a combination of the first few quantum numbers. For circular corrals, the wavefunctions are characterized by the principle and angular quantum numbers, $n, \ell$, respectively. $n-1$ defines the number of nodal lines in the radial direction from the center, while $\ell$ defines how many nodes occur angularly. For non-circular symmetric corrals, the angular momentum quantum number is not well-defined. However, the wave functions of circular, rectangular and triangular corrals exhibit alternation of sign and nodal line patterns that are reminiscent of nodal planes in atomic orbitals 30]. The lowest energy state has no nodal lines, the second lowest has one, etc. 35,36$]$. Based on these similarities, we refer to these states of the quantum corral as $s$-like and $p$-like, respectively. The nodal line pattern of a particular state of the quantum corral can be visualized by mapping the differential conductance at the energy corresponding to that state. In principle, the spin quantum number $m_{s}$ is also common between a 2D particle-in-a-box and a real atom, because $m_{s}$ only describes whether an electron has spin $+\frac{1}{2}$ or $-\frac{1}{2}$, and is a general property of electrons.

In contrast to $2 \mathrm{D}$ quantum corrals, three quantum numbers appear for real atoms. The magnetic quantum number is not present in 2D systems. However, as we show below, $p_{x^{-}}$ and $p_{y}$-like states do emerge in rectangular corrals [30]. Furthermore, the allowed values of the quantum numbers are different for quantum corrals and real atoms. For example, circular 2D quantum corrals feature 1p-type states (see Fig 1a,b), whereas in real atoms a $1 p$ state does not exist.

In addition to Fe atoms, a variety of other adsorbates can be used as scattering centers. Because of the ease and reliability with which they can be manipulated, $\mathrm{CO}$ molecules are 
often used [6, 37, 38. Carbon monoxide molecules on the $\mathrm{Cu}(111)$ surface are imaged as depressions with standard metallic tips 39 . A DFT study has suggested that this is due to destructive interference of the protruding orbital of oxygen atom with the states in the tip [40].

Throughout this document, we show the designs of various corrals and indicate copper atoms as orange dots and $\mathrm{CO}$ molecules as black dots with shading that represent the apparent size of the CO molecule as viewed in STM. Corral dimensions are reported in terms of the $\mathrm{Cu}(111)$ lattice constant $a=0.2556 \mathrm{~nm}$ 41.

\subsection{Tight binding description of dimers and trimers}

To create artificial dimers, we construct two connected corrals with an opening between them to accommodate coupling. Fig. 2(a) shows an example of a structure consisting of two coupled rectangular corrals.

The tight binding parameters of interest are the on-site energy, $\epsilon$, the nearest and next-nearest neighbor hopping parameters, $t_{1}$ and $t_{2}$ (not present for dimers) respectively, and the overlap integral, $s$ [42, 43], see Fig. 2. It was previously reported that the nextnearest-neighbor hopping integral can be non-negligible in artificial lattices $27,29,31,33$. To determine the magnitude of $t_{2}$, we also constructed and characterized trimers, see Fig. 2(c).

A tight binding calculation of a dimer, taking into account only the lowest energy state of each corral, results in the following expressions for the two states of the dimer

$$
\begin{aligned}
& E_{+}=\frac{\epsilon_{1}+t_{1}}{1+s} \\
& E_{-}=\frac{\epsilon_{1}-t_{1}}{1-s}
\end{aligned}
$$

where the subscript indicates the sign with which the states of the corral are added. The values of $E_{+}$and $E_{-}$can be directly extracted from differential conductance spec-

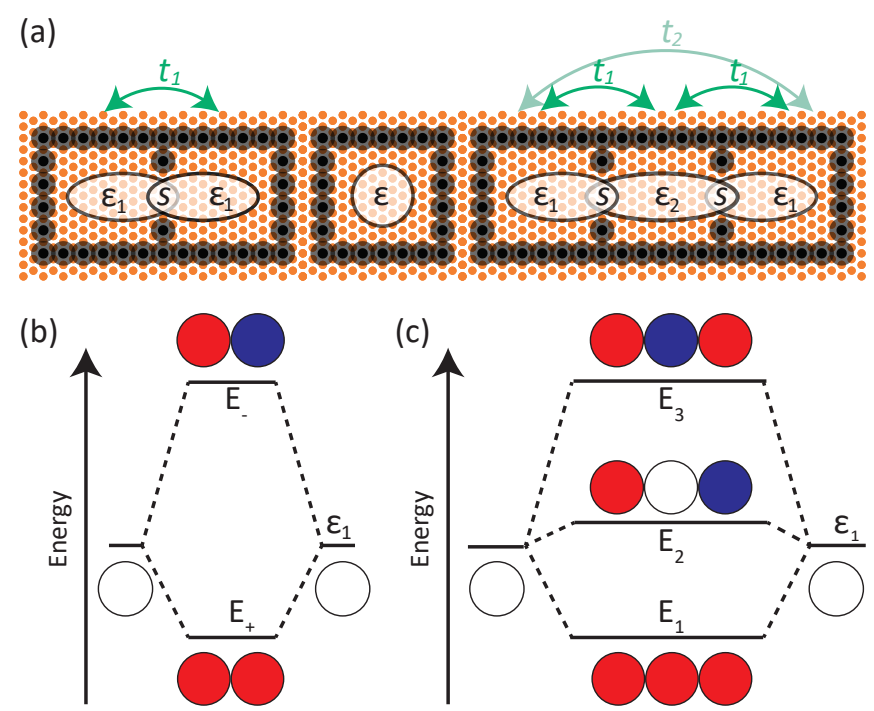

Figure 2: Coupling quantum corrals. (a) Example placements of CO molecules (black) on $\mathrm{Cu}(111)$ (orange) to produce a dimer, a lone corral and a trimer. White ovals roughly represent the spatial extent of the wave functions of the individual quantum corrals. (b) and (c) show the molecular orbital diagrams for a dimer and a trimer, respectively. Red represents a positive value of the wavefunction and blue negative. 
tra acquired at suitable positions above the dimer (taking the shape and extent of the wavefunction into account). Since the spatial confinement of the electrons in the dimer is different from those of isolated corrals (there is an extra available area when the barrier between two corrals is removed, the on-site energy is different for coupled and individual corrals. The resulting set of two equations with three unknowns (1) and 2) cannot be solved. To determine values of $\epsilon_{1}, t_{1}$ and $s$, we include calculations and measurements on a trimer, as represented in Fig. 2(c).

In the case of a trimer, there are three energy states that correspond to bonding, nonbonding and antibonding orbitals in molecules, as illustrated in Fig. 2(e). The energies of these three states are given by equations 3 , 4 and 5 , respectively.

$$
\begin{gathered}
E_{1}=\frac{\epsilon_{1}+\epsilon_{2}-4 s t_{1}+t_{2}-\sqrt{\left(-\epsilon_{1}-\epsilon_{2}+4 s t_{1}-t_{2}\right)^{2}-4\left(1-2 s^{2}\right)\left(\epsilon_{1} \epsilon_{2}-2 t_{1}^{2}+\epsilon_{2} t_{2}\right)}}{2\left(1-2 s^{2}\right)} \\
E_{2}=\epsilon_{1}-t_{2} \\
E_{3}=\frac{\epsilon_{1}+\epsilon_{2}-4 s t_{1}+t_{2}+\sqrt{\left(-\epsilon_{1}-\epsilon_{2}+4 s t_{1}-t_{2}\right)^{2}-4\left(1-2 s^{2}\right)\left(\epsilon_{1} \epsilon_{2}-2 t^{2}+\epsilon_{2} t_{2}\right)}}{2\left(1-2 s^{2}\right)}
\end{gathered}
$$

where $t_{2}$ is the next-nearest-neighbor hopping parameter, $\epsilon_{1}$ is the on-site energy of each of the outer two atoms (the same as in the dimer) and $\epsilon_{2}$ is the on-site energy of the central atom, see Fig. 2(c). Since $E_{1}, E_{2}$ and $E_{3}$ are also observable in experiment, we now have a system of five equations (1) to 5) and five unknowns. This allows us to obtain all tight binding parameters $\epsilon_{1}, \epsilon_{2}, s, t_{1}$, and $t_{2}$.

\section{Methods}

All experiments were performed at $T \approx 4.5 \mathrm{~K}$ in ultra-high vacuum with a ScientaOmicron LT-STM. A $\mathrm{Cu}(111)$ surface was prepared by several repetitions of sputtering with $\mathrm{Ar}^{+}$ and annealing at $550^{\circ} \mathrm{C}$. Carbon monoxide was leaked into the microscope chamber with a direct line of sight onto the $\mathrm{Cu}(111)$ crystal mounted in the microscope head to achieve a suitable coverage. Manipulation of carbon monoxide molecules was performed in feedback with a bias voltage of $20 \mathrm{~mA}$ and a current setpoint of approximately $50 \mathrm{nA}$, depending on the configuration of the tip apex. STM images were acquired in constant current mode. Differential conductance spectra and maps were acquired with the tip at constant height and using a standard lock-in amplifier technique. The frequency and amplitude of the applied modulation was $271 \mathrm{~Hz}$ and $10 \mathrm{mV}$ r.m.s. respectively. Integration time for signal acquisition was $50 \mathrm{~ms}$ during spectra and $20 \mathrm{~ms}$ during maps. All differential conductance spectra shown have been averaged over several measurements acquired at the same position, and divided by an average of several spectra taken on bare $\mathrm{Cu}(111)$ with the same tip apex to minimise the LDOS contribution from the tip [26].

Muffin tin calculations were performed to corroborate and supplement the experimental data. This technique is well-established, and has been used before to simulate results on the $\mathrm{CO} / \mathrm{Cu}(111)$ platform with reasonable accuracy $[27,29,31,33$. 


\section{Results}

\subsection{Individual Corrals}

We first characterize rectangular corrals. Note that because of the triangular symmetry of the underlying substrate, it is not possible to build perfectly square corrals. Fig. $3 \mathrm{a}$ shows the schematic structure and $\frac{d I}{d V}$ spectra of a rectangular corral with size $8 \sqrt{3} a \times 14 a$. Spectra taken at different positions exhibit peaks at different positions, corresponding to specific eigenstates. For example, the lowest energy level (approximately $-0.3 \mathrm{~V}$ ) has the highest local density of states (LDOS) in the center of the corral (black), whereas the next highest energy level $(-0.1 \mathrm{~V})$ is mainly observed away from center (at red, blue and green sites). The differential conductance map reveal the spatialextent of these states, see the top row in Fig. 3b. The corresponding simulated maps are shown in the bottom row of the same figure. In the case of degenerate levels, the modulus squared of the relevant eigenfunctions were summed. The simulations are in excellent agreement with the experimental observations.

For rectangular quantum corrals, there are two quantum numbers that determine the energy of the system and the shape of the wavefunction; $n_{x}$ and $n_{y}$. By comparing the experimental data to the results of the particle-in-a-box model, we can assign wave functions to the differential conductance maps and peaks in differential conductance spectra. For
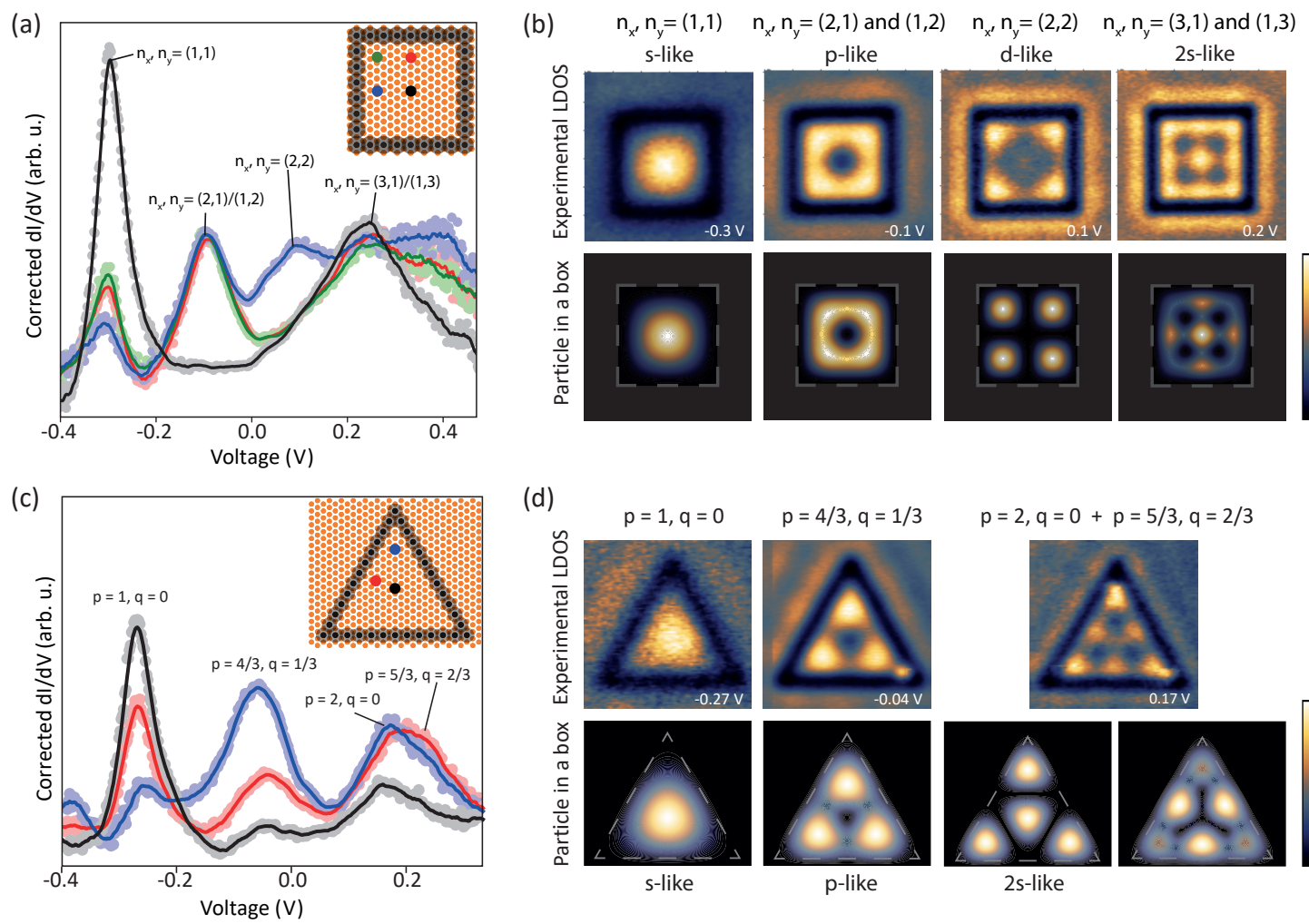

(d)

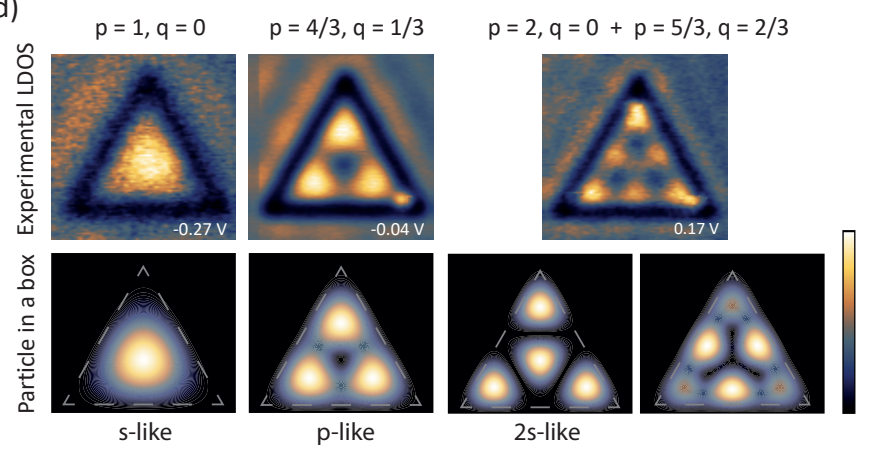

Figure 3: Differential conductance measurements on rectangular and triangular quantum corrals. (a) dI/dV spectroscopy acquired at the positions marked in the inset figure. (b) Top: experimental differential conductance maps (of size $6 \mathrm{~nm} \times 6 \mathrm{~nm}$ ) acquired at the energies stated; bottom: $|\Psi|^{2}$ calculated according to the particle in a box model. The quantum numbers are labeled above each peak in the $\mathrm{dI} / \mathrm{dV}$ and above each LDOS map. (c), (d) same as (a) and (b) but for triangular quantum corral. The images depict an area of $6.25 \mathrm{~nm} \times 6.25 \mathrm{~nm}$. 
the first few energy levels, we may draw an analogy to real atoms based on the number of nodal lines in $\Psi$ that intersect the center of the corral. The $n_{x}=1, n_{y}=1$ (no nodal lines) resembles an atomic $s$-orbital. Similarly, the $n_{x}=1, n_{y}=2\left(\right.$ and $n_{x}=2, n_{y}=1$ ) (one nodal line) and $n_{x}=2, n_{y}=2$ (two nodal lines), have a similar nodal line structure as $p$ - and $d$-type orbitals in atoms. The next highest state is the $2 s$-like state.

We now apply the same procedure to triangular corrals. An equilateral triangular corral is constructed with side lengths $12 \sqrt{3} a \cdot \frac{d I}{d V}$ spectroscopy was conducted at different positions, see Fig. 33. The lowest energy peak is observed at approximately $-0.27 \mathrm{eV}$ and has the highest amplitude in the center of the corral ( $s$-like). state. The second energy level is mainly localized near the corners ( $p$-like orbital). $\frac{d I}{d V}$ maps acquired at the peaks observed in the $\frac{d I}{d V}$ spectra are shown in Fig. $3 \mathrm{~d}$. For a particle-in-a-triangular-box, there are two quantum numbers; $p$ and $q$. The calculated eigenfunctions corresponding to the first four energy levels are shown in the bottom row of Fig. 3b. For the first two states, there is excellent agreement between experimental and simulated maps. The energy difference between the third $(p=2, q=0)$ and fourth $(p=5 / 3, q=2 / 3)$ lowest energy states of a particle in a triangular box is small. Consequently, both states contribute to the experimentally observed contrast at $V=0.17 \mathrm{~V}$.

\subsection{Corral size and on-site energy}

We now consider how altering the size of a corral affects the energies of the lowest levels, i.e. the on-site energies. We first focus on rectangular corrals. Fig. 4a shows a series of rectangular quantum corrals that were constructed. Differential conductance spectra acquired at the centers of the corrals are shown in Fig. 4 $\mathrm{b}$. As the corral is reduced in size, the ground state shifts to higher energies. The second peak for S1 at higher energies corresponds to a $2 s$-like orbital, vide infra. Note that peaks become progressively broader with increasing energy. We attribute this to two factors. First, the scattering potential of the $\mathrm{CO}$ molecules is finite $(0.9 \mathrm{eV}$ with respect to the onset of the surface state band when a radius of $0.3 \mathrm{~nm}$ is used). Hence, electrons with higher energy effectively experience a lower barrier height. Secondly, the number of CO molecules per unit area is larger for smaller corals, resulting in an increased coupling between surface and bulk states [44].

To rationalize the experimental observations, we model our system using a particle-ina-box model with finite potential barriers [36]. For a 2-dimensional rectangular box with finite barriers, the energies are given by

$$
E=\frac{2 \hbar^{2}}{m^{*}}\left(\frac{u_{n_{x}}^{2}}{L_{x}^{2}}+\frac{u_{n_{y}}^{2}}{L_{y}^{2}}\right)
$$

where $m^{*}=0.42 m_{e}$, the effective mass of the $\mathrm{Cu}(111)$ surface state electrons, and $L_{x}$ and $L_{y}$ correspond to the length of the box in the $x$ and $y$ direction, respectively. The variables $u_{n_{x}}$ and $u_{n_{y}}$, take the role of quantum numbers for which the following expressions hold

$$
u_{n_{i}}=\sqrt{u_{0}^{2}-v_{i}^{2}}=\left\{\begin{array}{l}
v_{i} \tan \left(v_{i}\right) \\
-v_{i} \cot \left(v_{i}\right)
\end{array}\right.
$$

with $u_{0}=\frac{\sqrt{2 m^{*} V_{0}} L_{i}}{2 \hbar}, v_{i}=\frac{\sqrt{2 m^{*}\left(V_{0}-E\right)} L_{i}}{2 \hbar}$ and $u_{i}=\frac{\sqrt{2 m^{*} E} L_{i}}{2 \hbar}$.

No analytical solutions exist for these equations and one has to rely on graphical or numerical methods. For a given $V_{0}, L_{i}$ and effective mass, the values of $u_{n_{i}}$ are fixed, and can be thought of as analogous to the quantum number in the energy equation that describes a particle in a $2 \mathrm{D}$ rectangular box with infinite barriers. To calculate the values of $u_{n}$, we use $V_{0}=0.9 \mathrm{eV}$ 27, 29, 31, 33 and $m^{*}=0.42 m_{e}$ [45, 46]. The values of $L_{x}$ and 

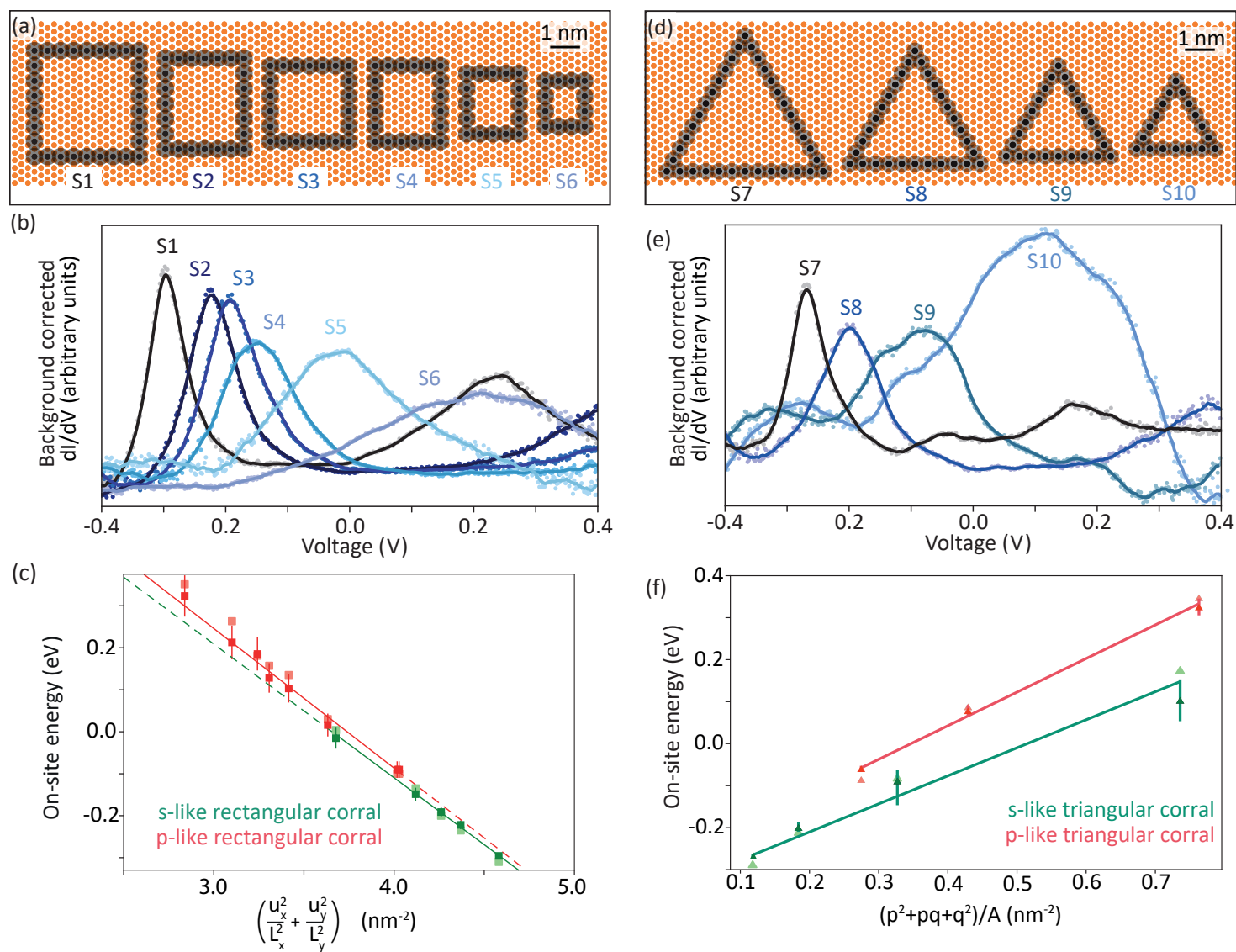

Figure 4: (a) Geometries of the rectangular corrals investigated. (b) $\frac{d I}{d V}$ spectra taken at the centers of the rectangular corrals shown in (a). (c) On-site energy as a function of $u_{n_{x}}^{2} / L_{x}^{2}+u_{n_{y}}^{2} / L_{y}^{2}$. Green and red points represent experimental data for $s$ - and $p$ - like states respectively. Light red and light green points represent the energies calculated using a muffin tin model. Solid lines represent a linear fit to the experimental data. (d)-(f) Same as (a)-(c) but for triangular corrals.

$L_{y}$ are determined by assuming that the dimensions of the boxes are defined by the edges of the CO molecules which have a diameter of $0.6 \mathrm{~nm}[27,29,31,33]$.

Figure 4 c shows a plot of the on-site energy versus $u_{n_{x}}^{2} / L_{x}^{2}+u_{n_{y}}^{2} / L_{y}^{2}$ for the lowest and second lowest states. Dark (light) green and red (light red) correspond to experimental (muffin tin) data of $s$-and $p$-like states, respectively. The experimental energies were determined by fitting Gaussian curves to each peak and finding the centers. The muffin tin-derived energies were calculated with the aforementioned values for $V_{0}, m^{*}$ and $\mathrm{CO}$ diameter. For both states, the energy depends linearly on $u_{n_{x}}^{2} / L_{x}^{2}+u_{n_{y}}^{2} / L_{y}^{2}$. From the gradient, we determine the effective electron masses to be $0.48 \pm 0.01 \mathrm{~m}_{e}$ and $0.46 \pm 0.01 \mathrm{~m}_{e}$ for the $s$-like and $p$-like states, respectively. These values are close to the effective electron mass of the unconfined surface state electrons.

An effective mass close to the mass of the $\mathrm{Cu}(111)$ surface state electrons suggests that the behavior of the electrons is dominated by the confinement induced by the CO molecules and that the interaction between the electrons and the periodic potential generated by the $\mathrm{Cu}$ surface can to first order be neglected. A small offset is visible between the lines for the $s-$ and $p$-like data, which we attribute to the fact that the confining potential is effectively lower for higher energy states.

We applied a similar procedure to triangular corrals. Figure 4 $\mathrm{d}$ shows the geometry 
of several triangular corrals that were realized, and Fig. 4p shows spectra acquired at the centres. The states of triangular corrals shift to higher energies the smaller the corral becomes. The data can be rationalized using a particle-in-a-box model using infinite barriers (analytical solutions for the finite barrier case are not available). The energy eigenvalues of a particle in an equilateral triangular box are given by

$$
E_{p, q}=\frac{h^{2}}{2 \sqrt{3} m^{*} A}\left(p^{2}+p q+q^{2}\right)
$$

where $p, q$ are the quantum numbers, $h$ is Planck's constant, $m^{*}$ is the effective electron mass and $A$ is the area of the triangle $35,47,48$. As shown in Fig. 4f, the experimentally determined on-site energy depends linearly on the inverse surface area, in agreement with equation (8).

\subsection{Coupling Corrals}

We now turn to coupled quantum corrals and show how tight-binding parameters can be extracted from experimental data. After a dimer is constructed (example shown in Fig. 5a), $\frac{d I}{d V}$ spectra are acquired on two positions. We do this to make use of the different spatial localization of the $E_{+}$and $E_{-}$states. Specifically, the anti-bonding $E_{-}$state has a node between the two corrals (the position denoted by an orange dot in the inset of Fig. 5 a). Only the bonding $E_{+}$state appears in the differential conductance spectrum taken at that site and we can fit the spectrum with a single Gaussian. Conversely, the anti-bonding $E_{-}$state has higher intensity at the outer regions of the dimer (red dot in Fig. 5 a).

Differential conductance maps were acquired at approximately the energies of the centers of each of the two peaks. The state at lower energy is delocalized over the entire structure, whereas the state at higher energy has a node between the two corrals. This is reminiscent of bonding and anti-bonding molecular orbitals, respectively.

Next, a trimer is constructed from the same-sized units as the dimer. To determine the experimental values of $E_{1}, E_{2}$ and $E_{3}$, we again exploit the different spatial distributions of these three states. Muffin tin calculations show that the intensity of the $E_{2}$ state is very low at the center corral. Hence, the two peaks in the differential conductance spectrum taken at this position (grey curve in Fig. 5d) can be assigned to $E_{1}$ and $E_{3}$, respectively. The obtained energies can then be used in the fitting procedure of the spectrum acquired at a corral at the end of the trimer (red curve in Fig. 5e and d). Taking these values and solving equations 1 to 5 results in the tight binding parameters listed in Table I.

Table 1: Tight binding parameters extracted from Fig. 5 .

\begin{tabular}{c|c} 
Parameter & Value \\
\hline$\epsilon_{1}$ & $-0.22 \pm 0.02 \mathrm{eV}$ \\
$\epsilon_{2}$ & $-0.23 \pm 0.01 \mathrm{eV}$ \\
$s$ & $0.5 \pm 0.3$ \\
$t_{1}$ & $-0.14 \pm 0.06 \mathrm{eV}$ \\
$t_{2}$ & $-0.02 \pm 0.03 \mathrm{eV}$ \\
\hline
\end{tabular}

The on-site energy of the individual corral of this size is $-0.19 \pm 0.02 \mathrm{eV}$, see Fig. 4t. We find an on-site energy of $-0.22 \pm 0.02 \mathrm{eV}$ and $-0.23 \pm 0.01 \mathrm{eV}$ for the sites in the dimer and central site in the trimer, respectively. This lowering of the on-site energy can be understood from the increased area that is available due to the removal of the CO molecules to couple the sites. Two CO molecules have been removed from the barrier, i.e. 


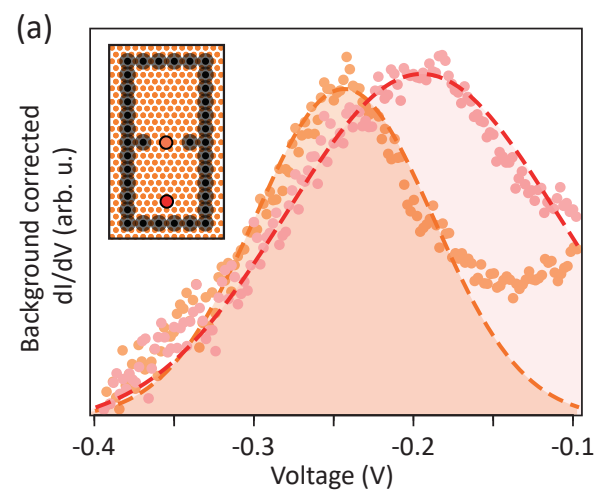

(b)

(c)
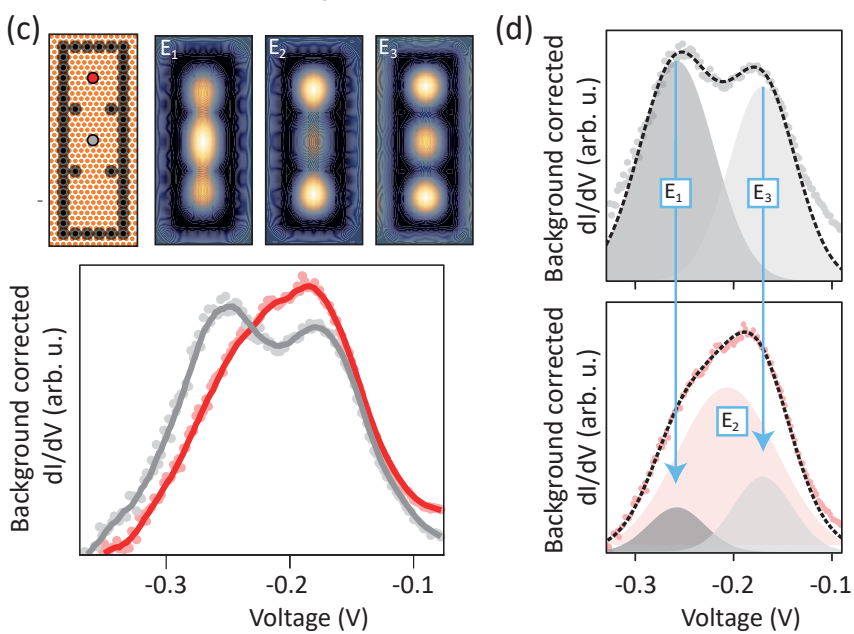

Figure 5: (a) $\frac{d I}{d V}$ spectra on two different positions of a dimer. Locations indicated by dots in the inset. (b) Differential conductance maps were acquired at the approximate energies where the maxima of the peaks lie. (c) Bottom: $\frac{d I}{d V}$ spectra acquired at the positions shown in corresponding colors in the top left diagram. LDOS maps (from muffin tin calculations) at $E_{1}=-0.26 \mathrm{eV}, E_{2}=-0.22 \mathrm{eV}, E_{3}=-0.18 \mathrm{eV}$, respectively. (d) Gaussian fitting procedure applied to the same two spectra to find the energies of interest. The shaded regions in each plot represent the individual Gaussians, which when summed, lead to the curves represented by dashed lines. The centers of the Gaussians correspond to $E_{1}$, $E_{2}$ and $E_{3}$ (labeled).

an additional area of $2 \times \pi(0.3)^{2}=0.56 \mathrm{~nm}^{2}$ is available for the electrons. The magnitude of the overlap integral, $s$, is significant and therefore must be included in tight binding parameters to yield accurate answers.

The same experiments and simulations were performed for coupling triangular corrals.

\subsection{Tuning parameters}

We now systematically investigate how the tight binding parameters depend on changing the gap width between corrals for both $s$ - and $p$-like states. For this, we created dimers out of rectangular quantum corrals with dimensions $6 \sqrt{3} a \times 10 a$ (same as in the previous section) and $8 \sqrt{3} a \times 14 a$. (Note that to calculate the area from these dimensions, the area that the $\mathrm{CO}$ molecules occupy must be subtracted). First, two corrals of equal size were constructed directly next to each other with the barrier fully closed; that is to say that the same barrier configuration that separates the two corrals separates the corrals from their surroundings. Fig. 6 a shows the schematic of a lone corral with dimension $8 \sqrt{3} a \times 14 a$, and the dimer with a full wall of $\mathrm{CO}$ molecules separating the corrals. The second column 


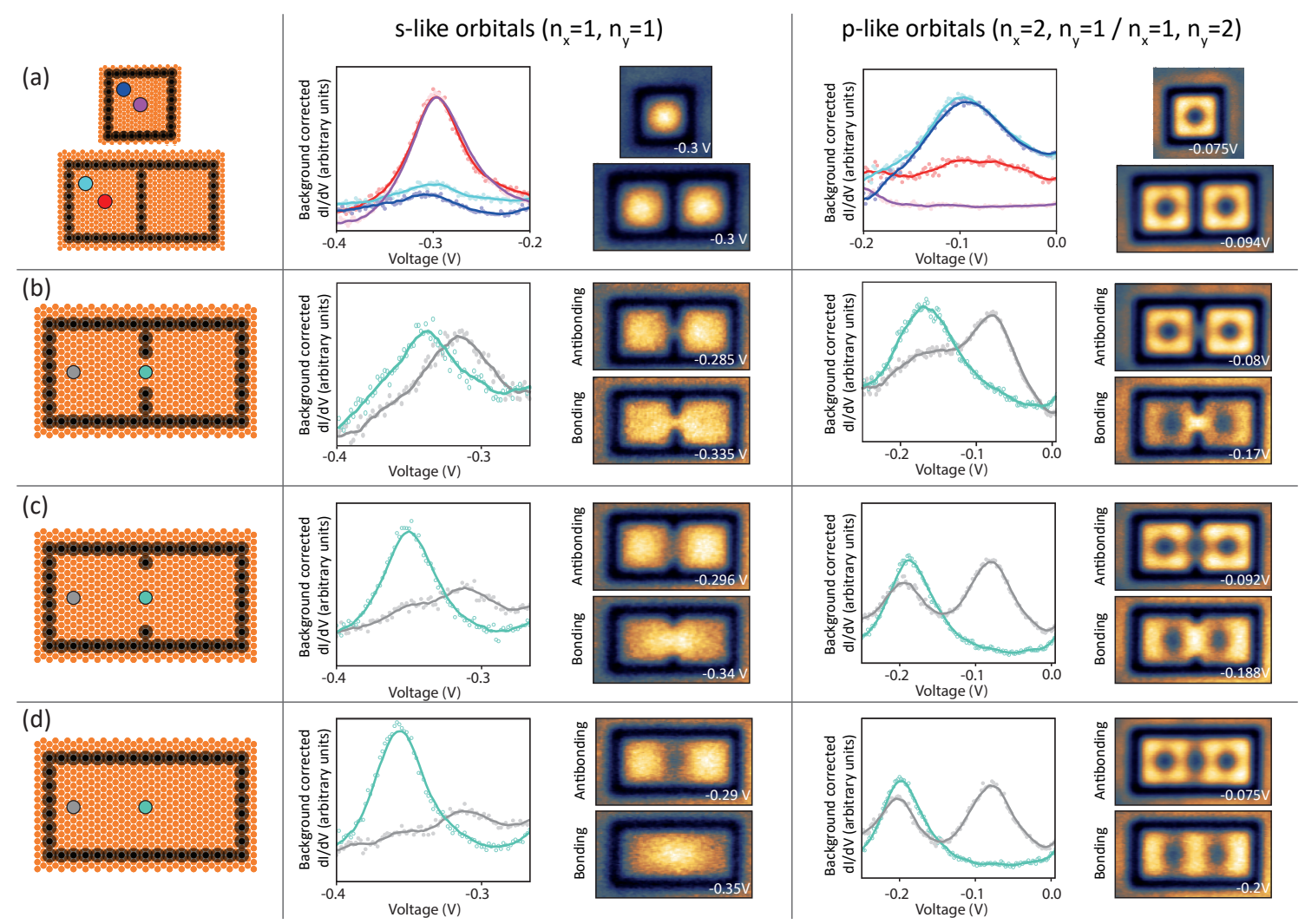

Figure 6: (a) From left to right, first the geometry of the coupled corral is shown. The second column demonstrates coupling of $s$-like orbitals: $\frac{d I}{d V}$ spectra acquired at the positions indicated by the color code and corresponding $\frac{d I}{d V}$ maps taken at the indicated energies. The third column focuses on coupling of $p$-like orbitals.

shows spectra taken at the positions marked in the designs. The peaks associated with the $s$-type orbitals occur at the same energy for the two systems, indicating that there is virtually no coupling between the corrals in the dimer with this barrier configuration (the hopping parameter is zero). The same observation is made for the $p$-type states (right hand side of the figure). This is significant because it has been assumed that coupling of electronic sites to the surrounding 2DEG plays a large role in broadening 31. The results reported here suggest that a "full barrier" would result in negligible coupling between states in a lattice and the surrounding 2DEG.

Next, CO molecules are removed from the center of the barrier, see Fig. 6b-d. As described before, $\frac{d I}{d V}$ spectra were acquired at the barrier between the corrals, and near the outer edge. By fitting Gaussian curves and finding their centers, we determine the energy level spacing between the bonding and anti-bonding states. Differential conductance maps were taken to verify the resemblance of these states to bonding and antibonding orbitals. The difference in energy between the two states increases with increasing gap width in the $\mathrm{CO}$ barrier between the two corrals. Furthermore, the states shift down in energy due to the effectively larger area that the electrons can occupy.

The most natural interpretation of the experimental data for the system without barrier, Fig. 6d, is to use a particle-in-a-rectangular-box model. In this picture, the lower energy state corresponds to the ground state with quantum numbers $n_{x}=1$ and $n_{y}=1$. The second state is the $n_{x}=2, n_{y}=1$ state, etc. However, it is also possible to interpret the results in the framework of two coupled quantum corrals. The lowest energy state of the rectangle can be thought of as the bonding combination of $s$-like orbitals of 

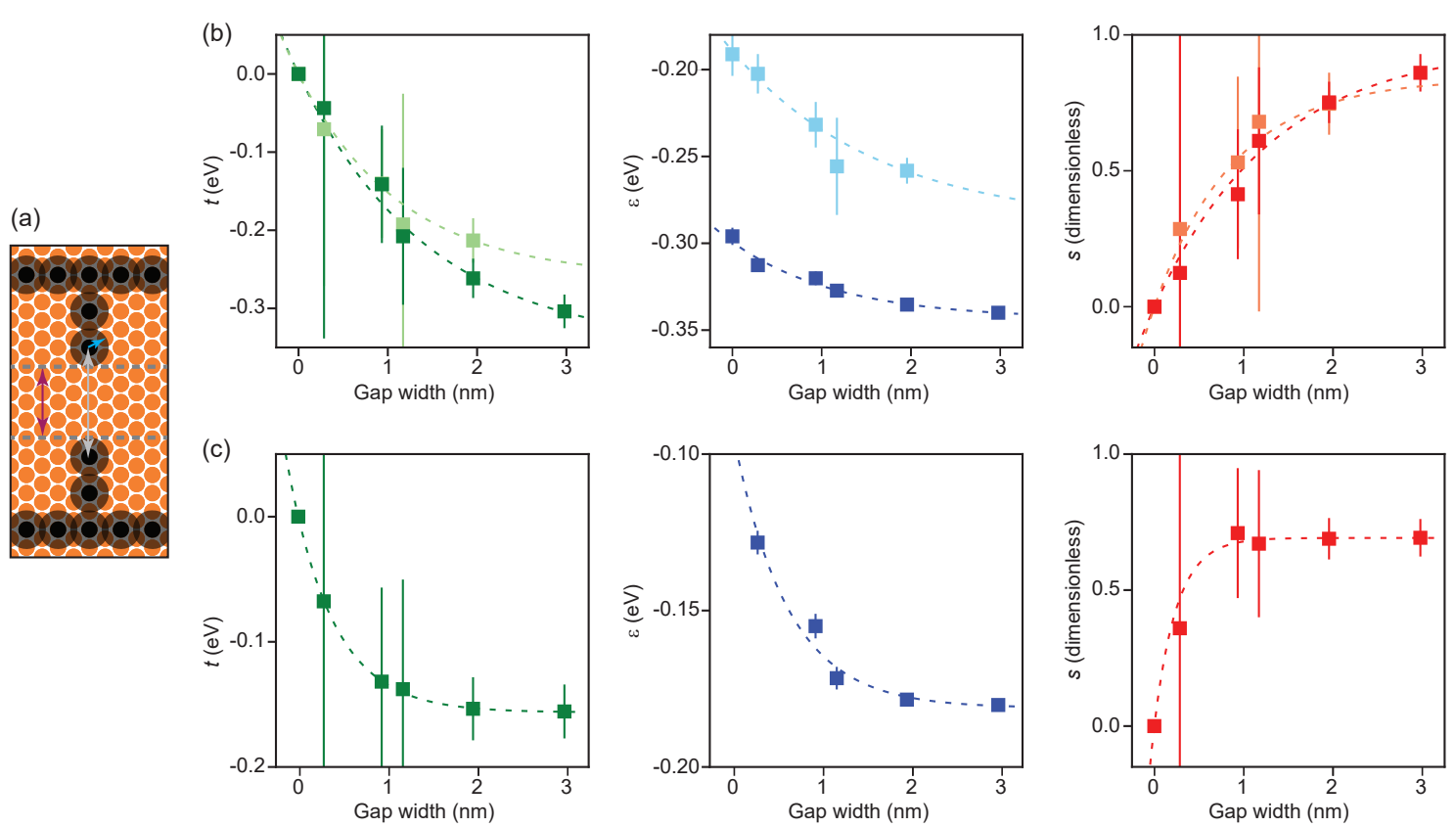

Figure 7: (a) The size of the gap in the barrier between the two corrals (red arrow) is the distance between the closest CO molecules in the barrier (gray arrow), minus two times the apparent size of the $\mathrm{CO}$ molecules (gray circle, blue arrow, $0.3 \mathrm{~nm}$ ). (b) From left to right: gap dependence of the hopping parameter, the on-site energy and overlap for $s$-like states, respectively. Dark and right colors represent data from rectangular corrals with sizes $8 \sqrt{3} a \times 14 a$ and $6 \sqrt{3} a \times 10 a$, respectively. (c) Same as (b) but now for $p$-like states.

the two quantum corrals. Similarly, the second lowest state would be the anti-bonding combination.

The bonding combination of the $p_{x}$-like states, where $x$ is the horizontal direction, shows vertical nodal lines at the centers of the individual corrals and enhanced intensity in the barrier region between the corrals (see right hand side of Fig. 6). The nodal line pattern of the map at higher energy can be rationalized by assuming that both the antibonding $p_{x}$-like state as well as the $p_{y}$-like state contribute to the contrast. The energy difference between $p$-like bonding and antibonding states is larger than for the $s$-like states.

Similar experiments were performed for coupled $6 \sqrt{3} a \times 10 a$ dimers (data not shown). From the available data on both corral sizes, tight binding parameters for coupling of both $s$-like and $p$-like states were derived. The results are shown in Fig. 7. The size of the gap in the barrier between the corrals is defined as the distance between the closest $\mathrm{CO}$ molecules of the barrier, minus two times the apparent radius of the $\mathrm{CO}$ molecules $(0.3$ nm, see Fig. $7 \mathrm{a}$ ). For both $s$ - and $p$-like states and for both corral sizes, the data points for the hopping parameter $(t)$, the on-site energy $(\epsilon)$, and the orbital overlap $(s)$ can be fitted with an exponential function (dotted lines). By tuning the gap width, the hopping parameter can be varied between $0 \mathrm{eV}$ and $\sim-0.3 \mathrm{eV}$ and $\sim-0.16 \mathrm{eV}$ for $s$ - and $p$-like states, respectively. We find that the on-site energy depends on the width of the gap in the barrier. The parameters depend more sensitively on gap width for the smaller corral. This can be rationalized from the additional area that becomes available to the confined electrons upon removing CO molecules (the relative increase in available area is larger for the smaller corral). Finally, the magnitude of the orbital overlap increases with gap width. Note that for unconfined electrons (infinite gap width) the overlap should be one. Fig. $7 \mathrm{~b}$ suggests that at least up to a gap width of $\approx 1.5 \mathrm{~nm}$, the hopping parameter and overlap 

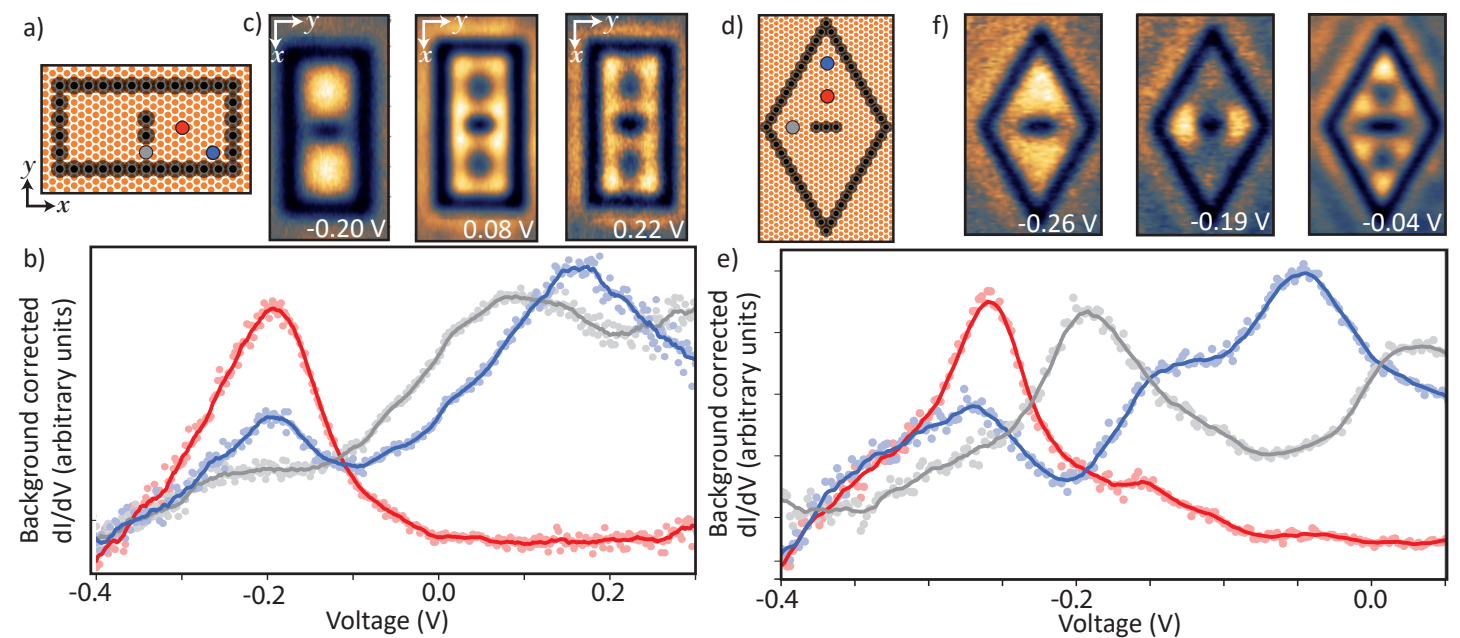

Figure 8: (a) Coupled rectangular corrals with a barrier that largely inhibits the coupling of $s$ and $p_{x}$-type states, while coupling of $p_{y}$-like states is clearly observed. $x$ and $y$ directions are specified in the figure. (b) Differential conductance spectra taken at the positions indicated in (a). (c) Differential conductance maps taken at the indicated energies. (d-f) same as (a-c) but for coupled triangular corrals.

are similar for the two different corral sizes.

\subsection{State selective coupling}

Since CO molecules can be removed selectively, it becomes possible to create geometries that allow coupling of $p$-type states only. Consider the geometries of coupled rectangular and triangular corrals shown in Fig. $8 \mathrm{a}$ and d. The amplitude of $s$-type wave functions is small at the position of the gaps in the barrier. Hence, coupling of $s$-type states should be small. In contrast, $p$-type states have significant amplitude at these positions and consequently these states should couple strongly. We first focus on the rectangular corrals. Fig. 8b shows differential conductance spectra taken at the positions indicated in Fig. 8a. A total of three peaks are observed. The amplitude of each peak differs from position to position. The peak at lowest energy corresponds to the ground state, i.e. it involves $s$-type states. At the energies corresponding to the $s$-type states, we only observe one peak, indicating that these states do not couple (coupling strength below the detection limit of our experiment). In contrast, the spectrum of the barrier region (gray) features a peak around $90 \mathrm{mV}$, whereas the spectrum taken at the corner of the corral (blue) has a peak at $170 \mathrm{mV}$. The corresponding differential conductance maps, Fig. 8c, reveal that the spatial extent of these states can be understood by considering coupling of $p_{y}$-type states. For the triangular corral, similar observations are made. This confirms the idea that artificial lattices allow coupling of one type of state only 49. Note that this provides a degree of freedom that is not available in real materials.

\subsection{Coupling corrals of different sizes}

Finally, we investigate the coupling of two corrals of different sizes, i.e. with different on-site energies for the $s$ - and $p$-like states. Fig. 9a shows the arrangement of such a polar dimer, with the barrier between corrals fully removed to maximize coupling. The $\frac{d I}{d V}$ spectra show the typical peaks associated with bonding and antibonding states. The corresponding differential conductance maps reveal that the lower (higher) energy state of 

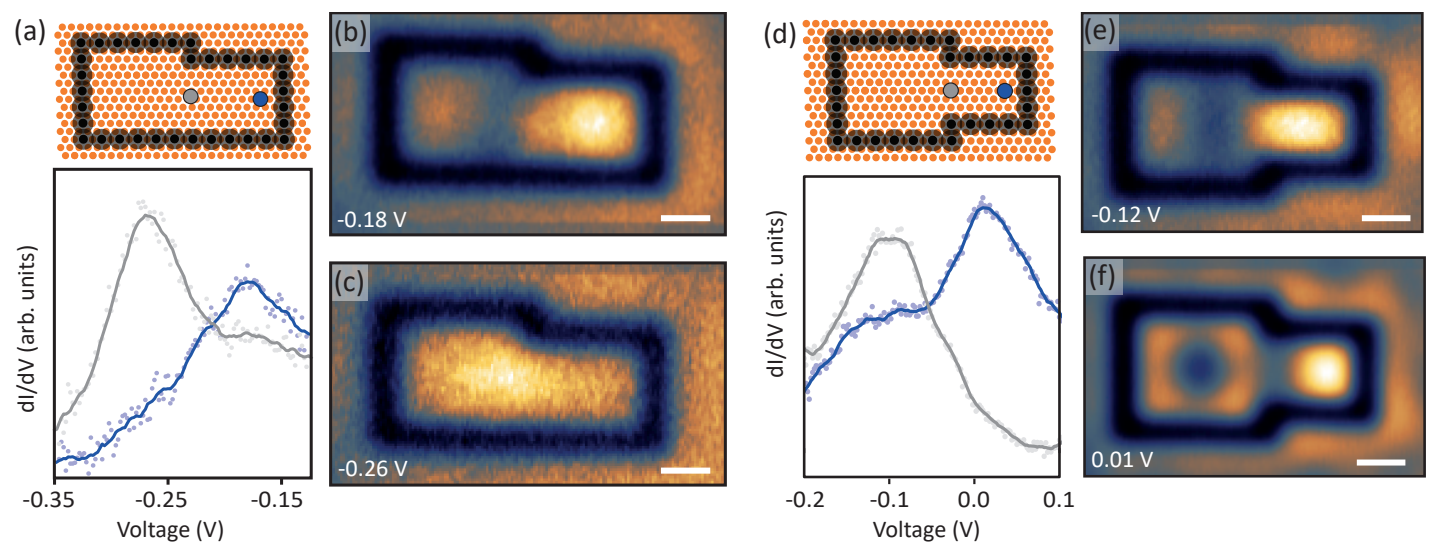

Figure 9: (a) Schematic structure of anisometric dimer consisting of a $6 \sqrt{3} a \times 12 a$ corral coupled to a $5 \sqrt{3} a \times 10 a$ corral. Differential conductance spectra acquired on positions highlighted in the inset. (b),(c) differential conductance maps of the two states observed in the $\frac{d I}{d V}$ spectra (energies indicated in the Figure). (d)-(f) same as (a-c), but now for a $6 \sqrt{3} a \times 12 a$ corral coupled to a $4 \sqrt{3} a \times 8 a$ corral. Inset scale bar (white) represents a length of $1 \mathrm{~nm}$

the dimer is primarily localized on the larger (smaller) corral, see Fig. 9b and c. This is in agreement with a tight binding model of a dimer with constituents with different on-site energy.

In general, electronic states couple if they spatially overlap and if they have a similar energy. Hence, if the sizes of the two corrals differ sufficiently, it is possible to couple the $s$-like state of a smaller corral with a $p$-like state of a larger corral. We therefore created a dimer consisting of a $6 \sqrt{3} a \times 12 a$ to a $4 \sqrt{3} a \times 8 a$ corral, see Fig. 9 . The $\frac{d I}{d V}$ spectra reveal two states with different spatial localization. The corresponding differential conductance maps show that the lower energy $s$-like state of the smaller corral couples with a $p$-like state of the larger corral. Similarly, the higher energy state can be thought of as an antibonding combination between $s$ - and $p$-like states (note the nodal line at interfaces between the two corrals).

\section{Conclusion}

To conclude, we have studied the coupling of rectangular and triangular quantum corrals into dimer and trimer structures. These shapes were chosen as they can be used as building blocks of artificial lattices. The electronic structure of the coupled corrals can be understood using a tight binding model also used for the coupling of atoms to molecules. Importantly, we investigated the available tight binding parameter space accessible with the $\mathrm{CO} / \mathrm{Cu}(111)$ platform, and showed how these parameters depend on the configuration of the coupled quantum corrals.

We first verified that the particle in a box model provides a good qualitative description of the electronic structure of rectangular and triangular quantum corrals. We determined the on-site energies of $s$ - and $p$ - like states of different sized corrals to confirm the relationship between on-site energy of the corral and box size. From this, we determined the effective masses of electrons in these systems to be on the order of $1 m_{e}$, significantly above the value for unconfined $\mathrm{Cu}(111)$ surface state electrons $\left(0.42 m_{e}\right)$.

We outlined a method to extract tight binding parameters (nearest and next near- 
est neighbor hopping parameters, overlap and on-site energy) by constructing dimers and trimers of corrals. By removing $\mathrm{CO}$ molecules from the barrier between corrals, exponential relationships were found between the tight binding parameters and the size of the gap in the barrier between the corrals. The hopping integral can be tuned between 0 and $-0.3 \mathrm{eV}$ and $-0.16 \mathrm{eV}$ for $s$ - and $p$-like states, respectively, by tuning the configuration of $\mathrm{CO}$ molecules in the barrier. In most cases, the overlap is not negligible and this term should be taken into account when modelling artificial molecules and lattices. Finally, we showed that in these coupled quantum corrals, one can control which states couple. For example, by appropriate placement of CO molecules coupling of $s$ - and $p_{x}$-like states can be inhibited, while allowing coupling of $p_{y}$-like states. Furthermore, it is possible to couple $s$ - and $p$-like states.

The results presented here are useful for future work on artificial lattices made using $\mathrm{CO}$ on $\mathrm{Cu}(111)$. A hypothetical lattice with certain desired coupling strengths and on-site energies can be designed by estimating the required unit size and barrier gap width from the trends reported here.

\section{Acknowledgements}

We thank Daniel Vanmaekelbergh and Jette van den Broeke for useful discussions.

Funding information We kindly acknowledge funding from the Dutch Research Council (NWO) via grant 16PR3245. .

\section{References}

[1] M. F. Crommie, C. P. Lutz and D. M. Eigler, Confinement of electrons to quantum corrals on a metal surface, Science 262(5131), 218 (1993), doi:10.1126/science.262.5131.218.

[2] C. R. Moon, L. S. Mattos, B. K. Foster, G. Zeltzer and H. C. Manoharan, Quantum holographic encoding in a two-dimensional electron gas, Nat. Nanotechnol. 4, 167 (2009).

[3] S. Loth, S. Baumann, C. P. Lutz, D. M. Eigler and A. J. Heinrich, Bistability in atomic-scale antiferromagnets, Science 335(6065), 196 (2012).

[4] F. E. Kalff, M. P. Rebergen, E. Fahrenfort, J. Girovsky, R. Toskovic, J. L. Lado, J. Fernández-Rossier and A. F. Otte, A kilobyte rewritable atomic memory, Nat. Nanotechnol. 11, 926 (2016).

[5] R. Achal, M. Rashidi, J. Croshaw, D. Churchill, M. Taucer, M. C. Taleana Huff, J. Pitters and R. A. Wolkow, Lithography for robust and editable atomic-scale silicon devices and memories, Nat. Commun. 9, 2778 (2018).

[6] A. J. Heinrich, C. P. Lutz, J. A. Gupta and D. M. Eigler, Molecule cascades, Science 298(5597), 1381 (2002).

[7] A. A. Khajetoorians, J. Wiebe, B. Chilian and R. Wiesendanger, Realizing allspin-based logic operations atom by atom, Science 332(6033), 1062 (2011). 
[8] M. Kolmer, R. Zuzak, G. Dridi, S. Godlewski, C. Joachim and M. Szymonski, Realization of a quantum hamiltonian boolean logic gate on the si(001):h surface, Nanoscale 7, 12325 (2015).

[9] T. Huff, H. Labidi, M. Rashidi, L. Livadaru, T. Dienel, R. Achal, W. Vine, J. Pitters and R. A. Wolkow, Binary atomic silicon logic, Nat. Electronics 1, 636 (2018).

[10] J. R. Hahn and W. Ho, Oxidation of a single carbon monoxide molecule manipulated and induced with a scanning tunneling microscope, Phys. Rev. Lett. 87, 166102 (2001).

[11] S.-W. Hla, L. Bartels, G. Meyer and K.-H. Rieder, Inducing all steps of a chemical reaction with the scanning tunneling microscope tip: Towards single molecule engineering, Phys. Rev. Lett. 85, 2777 (2000).

[12] I. Swart, L. Gross and P. Liljeroth, Single-molecule chemistry and physics explored by low-temperature scanning probe microscopy, Chem. Commun. 47, 9011 (2011).

[13] F. Albrecht, M. Neu, C. Quest, I. Swart and J. Repp, Formation and characterization of a molecule-metal-molecule bridge in real space, J. Am. Chem. Soc. 135, 9200 (2013).

[14] A. A. Khajetoorians, D. Wegner, A. Otte and I. Swart, Creating designer quantum states of matter atom- by-atom, Nat. Rev. Phys. 1, 703 (2019).

[15] W. Ko, C. Ma, G. D. Nguyen, M. Kolmer and A.-P. Li, Atomic-scale manipulation and in situ characterization with scanning tunneling microscopy, Advanced Functional Materials 29, 1903770 (2019).

[16] N. Nilius, T. M. Wallis and W. Ho, Development of one-dimensional band structure in artificial gold chains, Science 297, 1853 (2002).

[17] S. Fölsch, P. Hyldgaard, R. Koch and K. H. Ploog, Quantum confinement in monatomic cu chains on cu(111), Phys. Rev. Lett. 92, 056803 (2004).

[18] S. Fölsch, J. Yang, C. Nacci and K. Kanisawa, Atom-by-atom quantum state control in adatom chains on a semiconductor, Phys. Rev. Lett. 103, 096104 (2009), doi:10.1103/PhysRevLett.103.096104.

[19] B. Schuler, M. Persson, S. Paavilainen, N. Pavliček, L. Gross, G. Meyer and J. Repp, Effect of electron-phonon interaction on the formation of one-dimensional electronic states in coupled cl vacancies, Phys. Rev. B 91, 235443 (2015).

[20] J. Girovsky, J. L. Lado, F. E. Kalff, E. Fahrenfort, L. J. J. M. Peters, J. FernándezRossier and A. F. Otte, Emergence of quasiparticle bloch states in artificial crystals crafted atom-by-atom, SciPost Phys. 2, 020 (2017), doi:10.21468/SciPostPhys.2.3.020.

[21] R. Drost, T. Ojanen, A. Harju and P. Liljeroth, Topological states in engineered atomic lattices, Nature Physics 13, 668 (2018).

[22] S. R. Schofield, P. Studer, N. J. Hirjibehedin, C. F.and Curson, G. Aeppli and D. R. Bowler, Quantum engineering at the silicon surface using dangling bonds, Nat. Commun. 4, 1649 (2013).

[23] J. W. Park, H. S. Kim, T. Brumme, T. Heine and H. W. Yeom, Artificial relativistic molecules, Nat. Commun. 11, 815 (2020). 
[24] H. Kim, A. Palacio-Morales, T. Posske, L. Rózsa, K. Palotás, L. Szunyogh, M. Thorwart and R. Wiesendanger, Toward tailoring majorana bound states in artificially constructed magnetic atom chains on elemental superconductors, Science Advances 4(5) (2018), doi:10.1126/sciadv.aar5251.

[25] C. R. Moon, L. S. Mattos, B. K. Foster, G. Zeltzer, W. Ko and H. C. Manoharan, Quantum phase extraction in isospectral electronic nanostructures, Science 319(5864), $782(2008)$.

[26] K. K. Gomes, W. Mar, W. Ko, F. Guinea and H. C. Manoharan, Designer dirac fermions and topological phases in molecular graphene, Nature 483(7389), 306 (2012).

[27] M. R. Slot, T. S. Gardenier, P. H. Jacobse, G. C. van Miert, S. N. Kempkes, S. J. Zevenhuizen, C. M. Smith, D. Vanmaekelbergh and I. Swart, Experimental realization and characterization of an electronic lieb lattice, Nat. Phys. 13(7), 672 (2017).

[28] L. C. Collins, T. G. Witte, R. Silverman, D. B. Green and K. K. Gomes, Imaging quasiperiodic electronic states in a synthetic penrose tiling, Nat. Commun. 8, 15961 (2017).

[29] S. Kempkes, M. Slot, S. Freeney, S. Zevenhuizen, D. Vanmaekelbergh, I. Swart and C. M. Smith, Design and characterization of electrons in a fractal geometry, Nat. Phys. 15(2), 127 (2019).

[30] M. Slot, S. Kempkes, E. Knol, W. van Weerdenburg, J. van den Broeke, D. Wegner, D. Vanmaekelbergh, A. Khajetoorians, C. M. Smith and I. Swart, p-band engineering in artificial electronic lattices, Phys. Rev. X 9(1), 011009 (2019).

[31] S. Kempkes, M. Slot, J. van den Broeke, P. Capiod, W. Benalcazar, D. Vanmaekelbergh, D. Bercioux, I. Swart and C. M. Smith, Robust zero-energy modes in an electronic higher-order topological insulator, Nat. Mater. 18(12), 1292 (2019).

[32] G. A. Fiete and E. J. Heller, Colloquium: Theory of quantum corrals and quantum mirages, Rev. Mod. Phys. 75(3), 933 (2003), doi:10.1103/RevModPhys.75.933.

[33] S. E. Freeney, J. J. van den Broeke, A. J. J. H. van der Veen, I. Swart and C. M. Smith, Edge-dependent topology in Kekulé lattices, Phys. Rev. Lett. 124, 236404 (2020).

[34] R. W. Robinett, Visualizing the solutions for the circular infinite well in quantum and classical mechanics, Am. J. Phys. 64(4), 440 (1996).

[35] W.-K. Li and S. Blinder, Particle in an equilateral triangle: Exact solution of a nonseparable problem, J. Chem. Educ. 64(2), 130 (1987).

[36] D. J. Griffiths and D. F. Schroeter, Introduction to quantum mechanics, Cambridge University Press (2018).

[37] L. Bartels, G. Meyer and K.-H. Rieder, Basic steps involved in the lateral manipulation of single co molecules and rows of co molecules, Chem. Phys. Lett. 273(5), 371 (1997).

[38] R. J. Celotta, S. B. Balakirsky, A. P. Fein, F. M. Hess, G. M. Rutter and J. A. Stroscio, Invited article: Autonomous assembly of atomically perfect nanostructures using a scanning tunneling microscope, Rev. Sci. Inst. 85, 121301 (2014). 
[39] L. Bartels, G. Meyer and K.-H. Rieder, The evolution of co adsorption on cu (111) as studied with bare and co-functionalized scanning tunneling tips, Surf. Sci. 432(3), L621 (1999).

[40] A. Gustafsson and M. Paulsson, Scanning tunneling microscopy current from localized basis orbital density functional theory, Phys. Rev. B 93(11), 115434 (2016).

[41] S. Nakanishi and T. Horiguchi, Surface lattice constants of si (111), ni (111) and cu (111), Jpn. J. Appl. Phys. 20(3), L214 (1981).

[42] D. G. Pettifor and D. Pettifor, Bonding and structure of molecules and solids, vol. 193, Clarendon Press Oxford (1995).

[43] J. K. Burdett et al., Chemical bonding in solids, Oxford University Press (1995).

[44] S. Crampin, M. Boon and J. Inglesfield, Influence of bulk states on laterally confined surface state electrons, Phys. Rev. Lett. 73(7), 1015 (1994).

[45] P. O. Gartland and B. J. Slagsvold, Transitions conserving parallel momentum in photoemission from the (111) face of copper, Phys. Rev. B 12, 4047 (1975), doi:10.1103/PhysRevB.12.4047.

[46] L. Bürgi, L. Petersen, H. Brune and K. Kern, Noble metal surface states: Deviations from parabolic dispersion, Surface Science 447(1), L157 (2000).

[47] T. Kumagai and A. Tamura, Analysis of scanning tunneling microscopy images and scanning tunneling spectrum of electrons confined in equilateral triangular quantum corrals, J. Phys. Soc. Jpn. 77(1), 014601 (2007).

[48] G. Shaw, Degeneracy in the particle-in-a-box problem, J. Phys. A: Math. Nuc.Gen. $7(13), 1537$ (1974).

[49] M. R. Slot, S. N. Kempkes, E. J. Knol, W. M. Van Weerdenburg, J. J. Van Den Broeke, D. Wegner, D. Vanmaekelbergh, A. A. Khajetoorians, C. Morais Smith and I. Swart, P -Band Engineering in Artificial Electronic Lattices, Phys. Rev. X 9(1), 11009 (2019), doi:10.1103/PhysRevX.9.011009. 